\title{
Air Displacement Plethysmography for Estimating Body Composition Changes with Weight Loss in Middle-Aged Japanese Men
}

\author{
Hiroyuki Sasai ${ }^{a, b}$ Yoshio Nakata ${ }^{a}$ Miyuki Nemoto ${ }^{a}$ Kazunori Ohkawara ${ }^{b, c}$ \\ Tomoaki Matsuo $^{a}$ Maeng-Kyu Kim ${ }^{d}$ Yasutomi Katayama ${ }^{e}$ Kiyoji Tanaka ${ }^{a}$ \\ ${ }^{a}$ Graduate School of Comprehensive Human Sciences, University of Tsukuba, Ibaraki, \\ b Japan Society for the Promotion of Science, \\ ${ }^{\mathrm{c}}$ Health Promotion and Exercise Program, The National Institute of Health and Nutrition, Tokyo, \\ ${ }^{\mathrm{d}}$ Department of Medicine, Metabolism and Endocrinology, Juntendo University School of Medicine, Tokyo, \\ ${ }^{e}$ Faculty of Education, Kogakkan University, Mie, Japan
}

\section{Keywords}

Air displacement plethysmography .

Dual energy X-ray absorptiometry .

Percentage of fat mass - Weight loss - Obesity

\section{Summary}

Aim: To examine the degree to which air displacement plethysmography (ADP) can track body composition changes in response to weight loss in obese Japanese men. Method: 50 men, aged 30-65 years with a mean BMI of $30 \mathrm{~kg} / \mathrm{m}^{2}$, were included in a 3-month weight loss program. Percentage of fat mass (\%FM) was determined by dual energy X-ray absorptiometry (DXA) and ADP at baseline and month 3. Results: With $6.2 \pm 4.3 \mathrm{~kg}$ of weight loss, \%FM, as determined by DXA and ADP, significantly decreased by $3.9 \pm 2.9 \%$ and $3.9 \pm 3.3 \%$, respectively. There was no mean difference for change $(\Delta)$ in \%FM between the two methods. DXA-derived $\Delta \% \mathrm{FM}$ significantly correlated with $\triangle \% \mathrm{FM}$ determined by ADP $\left(R^{2}=0.48, p<0.01\right)$. Furthermore, the Bland-Altman plots demonstrated no systematic bias for $\Delta \% \mathrm{FM}(r=-0.20$, $p=0.17)$. However, \%FM by ADP $(r=0.42)$ at baseline and $\Delta \% F M$ by ADP $(r=-0.54)$ were significantly correlated to the differences between $\triangle \% F M$ by DXA and ADP. Conclusion: These results suggest that ADP is comparably accurate for evaluating $\triangle \% \mathrm{FM}$ when compared with DXA, although there were proportional biases.

\section{Introduction}

The obesity epidemic has become one of the biggest public health concerns worldwide, including Asian countries [1, 2], because of its close relationship to chronic diseases such as hypertension, dyslipidemia, diabetes and cardiovascular disease $[3,4]$. Since obesity is characterized and defined as the excess accumulation of body fat, accurate measurements of body composition are of utmost importance for public health and clinical perspectives. The ability of health professionals to accurately estimate body composition changes in obese individuals is also critical for determining the effectiveness of weight loss or management strategies.

Several body composition methods are available, which differ in terms of their theoretical bases and scientific assumptions as well as in terms of their cost, complexity and subject acceptability. For example, hydrostatic weighting (HW) has long been considered the gold standard for measuring body composition [5]. However, it requires many methodological assumptions and inconveniences that limit its usefulness and widespread application. Consequently, dual energy X-ray absorptiometry (DXA) has emerged as one of the most accurate techniques to assess body composition. DXA uses a threecompartment model to provide an estimate of bone mineral content, fat and lean soft tissue. DXA is easier to administer than HW and correlates well with other measures of body composition such as HW, total body water and multicompartment approaches [6, 7]. DXA can also detect body composition changes due to weight loss [8].

\begin{tabular}{ll}
\hline KARGER & $\oplus$ 2010 S. Karger GmbH, Freiburg \\
Fax +497614520714 & Accessible online at: \\
Information@Karger.de & www.karger.com/ofa \\
www.karger.com &
\end{tabular}


Air displacement plethysmography (ADP) has emerged as a fairly novel technique that provides a body composition estimate from body density using a two-compartment model, as does HW [9]. The biggest difference between ADP and HW is that ADP does not require participants to exhale maximally while submerged under water. Therefore, ADP seems much more feasible and comfortable than HW. Cross-sectional studies showed that percentages of fat mass (\%FM) estimated by ADP highly correlate with those from DXA $[10,11]$.

Only few studies have demonstrated that ADP was sensitive enough to detect, similarly to DXA, moderate body composition changes over various time periods (from 2 to 16 months) and percent weight loss (from 4.7 to $7.0 \%$ ), primarily among Caucasian adults [11-13]. Asians, including Japanese, have greater \%FM than Caucasians for the same BMI and age [14]. Therefore, it is important to determine whether ADP is a valid method of tracking body composition changes in Japanese men.

The purpose of this study was to examine whether ADP could accurately track body composition changes in response to weight loss in obese Japanese men, using DXA as a reference method. Better understanding of this issue will be useful for designing longitudinal studies regarding body composition as well as in providing clinically relevant information for health professionals.

\section{Participants and Methods}

\section{Participants}

We recruited participants from communities around the University of Tsukuba for 3-month weight loss programs through local newspaper advertisements and study flyers. Eligibility criteria included the following: i) men, ii) aged 30-65 years, iii) a BMI $>25 \mathrm{~kg} / \mathrm{m}^{2}$ according to the domestic obesity guideline [15] and iv) no history of cardiovascular disease. In Japan, despite the fact that only $2-3 \%$ has been characterized as having a $\mathrm{BMI} \geq 30 \mathrm{~kg} / \mathrm{m}^{2}$, the prevalence of metabolic disorders is relatively high $[16,17]$. Thus, the cutoff value for the definition of obesity for Asian populations was used [15]. After an orientation session, 54 men participated in the weight loss programs in 2007. The men were non-randomly assigned to receive either a dietary program $(n=30)$ or an exercise program $(\mathrm{n}=24)$. These two weight loss methods were employed to achieve a wide range of \%FM changes. We excluded four men with incomplete assessments after the programs. Thus, data from the remaining 50 men (dietary program: $n=26$; exercise program: $n=24$ ) were analyzed. We fully explained the purpose and design of the study to each participant before they gave written informed consent. The research protocol was approved by the institutional review board at the University of Tsukuba and thus meets the standards of the Declaration of Helsinki.

\section{Weight Loss Programs}

The dietary program consisted of weekly group-based 90-min instructional sessions for 3 months and of individual counseling by trained staff. In each session, the participants received lectures on low-calorie diets and eating behaviors. We instructed them to consume a well-balanced 1,680-kcal diet per day. The exercise program consisted of 90-min sessions of aerobic exercise three times per week for 3 months. Each exercise session began with 15-30 min of warm-up activities such as stretching. The session was followed by $30-60$ min of brisk walking performed outdoors in the campus of the University of Tsukuba and finished with 15-30 min of cool-down activities.

\section{Energy Intake and Expenditure}

Energy intake in kilocalorie of both weight loss programs was assessed before and during a 2 -week period (weeks 9 and 10) by 3 -day weighed dietary records, and dietary recall interviews for each participant were performed by skilled dieticians. The dietary data for each participant were analyzed by using commercially available computer software (Excel Eiyo-kun, Kenpakusha, Tokyo, Japan). Total energy expenditure was also assessed by a validated uniaxial accelerometer (Lifecorder-EX; Suzuken, Nagoya, Japan). The accelerometer was firmly attached to the participant's clothing (belt or waistband) during all waking hours (except while bathing and sleeping) 2 weeks before starting the 3 -month programs, for baseline examination, and throughout the programs. 42 men (23 in the dietary group and 19 in the exercise group) successfully completed the measurements described above.

\section{Measurement Procedures}

We instructed the participants not to participate in vigorous physical activity or alcohol consumption within $24 \mathrm{~h}$ prior to body composition measurement and to refrain from eating or drinking for $2 \mathrm{~h}$ prior to the measurements. The participants removed all metal objects and jewelry during the measurements. We assessed height to the nearest $0.1 \mathrm{~cm}$ using a wall-mounted stadiometer. Body weight was measured to the nearest $0.01 \mathrm{~kg}$ using DXA equipment. We then calculated BMI as body weight in kilograms divided by squared height in meters. Waist circumference was measured to the nearest $0.1 \mathrm{~cm}$ at the level of the umbilicus using a flexible retractable fiberglass tape measure. We always implemented DXA measurement first and then ADP within 30 min. All measurements were conducted in the same order at baseline and at month 3 .

\section{Dual Energy X-Ray Absorptiometry}

We measured \%FM by DXA (\%FM DXA $_{\text {f }}$ ) using a Lunar DPX-NT densitometer (Lunar, Madison, WI, USA). We calibrated the densitometer every day according to manufacturer's recommendations. The densitometer calculated soft tissue mass, including fat and lean tissue mass, from the ratio of mass attenuation coefficients ( $\mathrm{R}$ value) at $40-50 \mathrm{keV}$ and $80-100$ $\mathrm{keV}$. We defined fat-free mass as lean tissue mass plus bone mineral content. During each measurement, the participants remained motionless in the supine position while the scanning arm passed over his body in parallel 1-cm strips. To minimize technical error, the same examiner operated the densitometer and positioned the participant at baseline and month 3 . The coefficients of variations (CVs) in \%FM with repeated examinations were less than $2 \%$ in our laboratory $(n=33)$.

\section{Air Displacement Plethysmography}

We measured \%FM by ADP (\%FM $\mathrm{ADP}_{\text {) }}$ ) using a Bod Pod (Life Measurement Inc., Concord, CA, USA) according to manufacturer's instructions. The physical concepts and operational principles of ADP were described elsewhere [10]. Before each measurement, an examiner calibrated the Bod Pod six consecutive times to estimate the mean volume of the chamber with the 49.4901 cylinder. The participant wore trunks and a swim cap to minimize clothing and compress air, thereby estimating volume variations. Then, the participant was weighed on an electronic scale which was calibrated in advance with two standard $10-\mathrm{kg}$ weights. The participant sat quietly in the chamber while their raw body volume ( $\mathrm{Vb}$ ) was measured consecutively until two values within $150 \mathrm{ml}$ were obtained. We estimated thoracic gas volume (Vtg) by having participants perform the panting maneuver according to manufacturer's instructions until a successful measurement (the merit and airway value was less than 1 or $35 \mathrm{~cm} \mathrm{H}_{2} \mathrm{O}$ ) was obtained. Briefly, the participant wore a nose clip and breathed normally for three breathing cycles through a tube connected to the internal system. At the midpoint of an exhalation, the airway tube was momentarily occluded. And, the exam- 
Table 1. Comparison of body composition results obtained using DXA and ADP at baseline and month 3 among middle-aged obese Japanese men*

\begin{tabular}{|c|c|c|c|c|c|c|c|c|c|}
\hline & \multicolumn{3}{|c|}{ Dietary program $(n=26)$} & \multicolumn{3}{|c|}{ Exercise program $(n=24)$} & \multicolumn{3}{|c|}{ Combined $(n=50)$} \\
\hline & Baseline & Month 3 & Change & Baseline & Month 3 & Change & Baseline & Month 3 & Change \\
\hline Age, years & $47.1 \pm 7.9$ & & & $48.5 \pm 9.4$ & & & $47.8 \pm 8.6$ & & \\
\hline Height, cm & $170.1 \pm 5.1$ & & & $170.0 \pm 6.3$ & & & $170.1 \pm 5.6$ & & \\
\hline Body weight, kg & $87.6 \pm 11.2$ & $78.7 \pm 11.2$ & $-8.8 \pm 3.6^{\mathrm{e}}$ & $87.8 \pm 10.1$ & $84.4 \pm 9.2$ & $-3.4 \pm 2.9^{\mathrm{e}}$ & $87.7 \pm 10.6$ & $81.5 \pm 10.6$ & $-6.2 \pm 4.3^{\mathrm{e}}$ \\
\hline $\mathrm{BMI}, \mathrm{kg} / \mathrm{m}^{2}$ & $30.2 \pm 3.2$ & $27.1 \pm 3.1$ & $-3.1 \pm 1.3^{\mathrm{e}}$ & $30.4 \pm 3.4$ & $29.2 \pm 3.0$ & $-1.2 \pm 1.0^{\mathrm{e}}$ & $30.3 \pm 3.2$ & $28.1 \pm 3.2$ & $-2.2 \pm 1.5^{\mathrm{e}}$ \\
\hline Overweight $^{\mathrm{a}}, \%$ & 46.2 & 53.8 & & 58.3 & 62.5 & & 52.0 & 58.0 & \\
\hline Obese $^{\mathrm{b}}, \%$ & 53.8 & 15.4 & & 41.7 & 33.3 & & 48.0 & 24.0 & \\
\hline Waist circumference, $\mathrm{cm}$ & $102.1 \pm 7.8$ & $92.9 \pm 8.8$ & $-9.2 \pm 4.5^{\mathrm{e}}$ & $102.4 \pm 7.8$ & $97.9 \pm 6.9$ & $-4.5 \pm 2.8^{\mathrm{e}}$ & $102.3 \pm 7.7$ & $95.3 \pm 8.3$ & $-7.0 \pm 4.4^{\mathrm{e}}$ \\
\hline Fat-free mass ${ }^{c}, \mathrm{~kg}$ & $57.1 \pm 6.4$ & $55.4 \pm 5.7$ & $-1.7 \pm 1.6^{\mathrm{e}}$ & $58.8 \pm 5.5$ & $58.5 \pm 5.1$ & $-0.3 \pm 1.5$ & $57.9 \pm 5.9$ & $56.9 \pm 5.6$ & $-1.0 \pm 1.7^{\mathrm{e}}$ \\
\hline Bone mass ${ }^{\mathrm{c}}, \mathrm{g}$ & $3046 \pm 475$ & $3065 \pm 466$ & $19 \pm 126$ & $3074 \pm 363$ & $3113 \pm 394$ & $39 \pm 113$ & $3060 \pm 421$ & $3088 \pm 429$ & $29 \pm 119$ \\
\hline Body volume $^{\mathrm{d}}, 1$ & $85.5 \pm 11.6$ & $75.8 \pm 11.7$ & $-9.7 \pm 4.1^{\mathrm{e}}$ & $85.1 \pm 10.7$ & $81.1 \pm 9.6$ & $-4.1 \pm 3.2^{\mathrm{e}}$ & $85.3 \pm 11.0$ & $78.3 \pm 10.9$ & $-7.0 \pm 4.6^{\mathrm{e}}$ \\
\hline Body density ${ }^{\mathrm{d}}, \mathrm{kg} / \mathrm{l}$ & $1.02 \pm 0.01$ & $1.03 \pm 0.01$ & $0.01 \pm 0.01^{\mathrm{e}}$ & $1.03 \pm 0.01$ & $1.03 \pm 0.01$ & $0.01 \pm 0.01^{\mathrm{e}}$ & $1.02 \pm 0.01$ & $1.03 \pm 0.01$ & $0.01 \pm 0.01^{\mathrm{e}}$ \\
\hline \multicolumn{10}{|l|}{$\% \mathrm{FM}$} \\
\hline DXA & $34.5 \pm 4.4$ & $29.1 \pm 6.0$ & $-5.4 \pm 3.0^{\mathrm{e}}$ & $32.8 \pm 4.1$ & $30.4 \pm 4.9$ & $-2.4 \pm 1.8^{\mathrm{e}}$ & $33.7 \pm 4.3$ & $29.7 \pm 5.4$ & $-3.9 \pm 2.9^{\mathrm{e}}$ \\
\hline $\mathrm{ADP}$ & $34.9 \pm 4.6$ & $29.7 \pm 5.4$ & $-5.2 \pm 3.3^{\mathrm{e}}$ & $31.8 \pm 4.6$ & $29.2 \pm 4.8$ & $-2.6 \pm 2.8^{\mathrm{e}}$ & $33.4 \pm 4.8$ & $29.5 \pm 5.1$ & $-3.9 \pm 3.3^{\mathrm{e}}$ \\
\hline $\begin{array}{c}\Delta \text { between DXA } \\
\text { and ADP }\end{array}$ & $-0.4 \pm 2.8$ & $-0.6 \pm 2.6$ & $-0.2 \pm 2.2$ & $1.0 \pm 3.0$ & $1.2 \pm 3.0$ & $0.2 \pm 2.8$ & $0.3 \pm 2.9$ & $0.2 \pm 2.9$ & $0.0 \pm 2.5$ \\
\hline
\end{tabular}

$\Delta=$ Differences.

$*$ Values are means $\pm \mathrm{SD}$.

${ }^{\mathrm{a}} \mathrm{BMI}$ of $25-30 \mathrm{~kg} / \mathrm{m}^{2}$.

${ }^{\mathrm{b}} \mathrm{BMI}$ of greater than $30 \mathrm{~kg} / \mathrm{m}^{2}$.

${ }^{\mathrm{C}}$ Measured by DXA.

${ }^{\mathrm{d}}$ Measured by ADP.

'Significantly different from baseline.

iner signaled him to perform three small puffs of air into the tube while maintaining a tight seal around the end of the tube. We used the measured $\mathrm{Vtg}$ to calculate a corrected $\mathrm{Vb}$ (raw $\mathrm{Vb}$ minus $\mathrm{Vtg}$ ) and computed body density as body weight divided by the corrected $\mathrm{Vb}$. We calculated $\% \mathrm{FM}_{\mathrm{ADP}}$ using Siri's equation [18]. The CVs in \%FM with repeated examinations were less than $5 \%$ in our laboratory $(n=8)$.

\section{Statistical Analysis}

We chose DXA, which is not a recognized gold standard, as the reference method as it has been considered a reasonable alternative to a multicompartment approach [8]. After stratifying by program, we applied a paired Student's t-test to compare the mean \%FM from DXA and ADP at each time point and to assess the difference between measurements at baseline and month 3. A simple linear regression analysis examined the proportion of $\% \mathrm{FM}_{\mathrm{DXA}}$ and $\% \mathrm{FM}_{\mathrm{ADP}}$ at each time point. Bland-Altman plots [19] assessed the potential bias between DXA and ADP at each time point in wide ranges of body composition and body composition changes $(\Delta)$. We tested the null hypothesis that the slope was equal to zero in the models to consider the presence of bias. In addition, Pearson's correlation coefficients were calculated to explain the variance of the difference between $\Delta \% \mathrm{FM}$ by DXA and ADP. A p value less than 0.05 was regarded as statistically significant. We performed all statistical analyses using SAS, version 9.1 (SAS Institute, Inc., Cary, NC, USA).

\section{Results}

Table 1 describes baseline characteristics and compares body composition results obtained using DXA and ADP at base-
Table 2. Summary of simple regression analysis and Bland-Altman approach for \%FM by ADP at baseline and month 3 compared to DXA

\begin{tabular}{lcc}
\hline & Baseline & Month 3 \\
\hline Simple regression analysis & & \\
$\quad$ Slope & 0.71 & 0.91 \\
$\quad$ Intercept & 9.97 & 2.94 \\
$\mathrm{r}$ & 0.79 & 0.85 \\
$\mathrm{R}^{2}$ & 0.63 & 0.72 \\
SEE & 2.62 & 2.92 \\
$\mathrm{P}$ & $<0.01$ & $<0.01$ \\
\hline Bland-Altman approach & & \\
Bias & & \\
95\% LoA & $\mathrm{b}$ & 0.25 \\
$\mathrm{r}$ & 0.25 & $-5.48,5.98$ \\
$\mathrm{p}$ & $-5.52,6.02$ & 0.13 \\
\hline
\end{tabular}

SEE $=$ Standard error of estimate; $\mathrm{LoA}=$ limits of agreement. ${ }^{\mathrm{a} M e a n}$ difference between DXA and ADP (i.e., DXA minus ADP).

${ }^{b}$ Upper and lower $95 \%$ LoA.

line and month 3. Attendance rates were $77.5 \%$ for dietary sessions and $86.8 \%$ for exercise sessions. The weight loss programs significantly reduced body weight, waist circumference, body volume and \%FM, and significantly increased body density in both programs and in the combined data. Fat-free mass 
Fig. 1. Simple regression analysis for changes $(\Delta)$ in $\% \mathrm{FM}$ determined by DXA and ADP. The bold solid line indicates the regression line between $\triangle \% \mathrm{FM}$ from DXA and ADP. The dashed lines represent $95 \%$ prediction intervals. @: participants engaged in dietary program, $\bigcirc$ : participants engaged in exercise program, SEE: standard error of estimate.

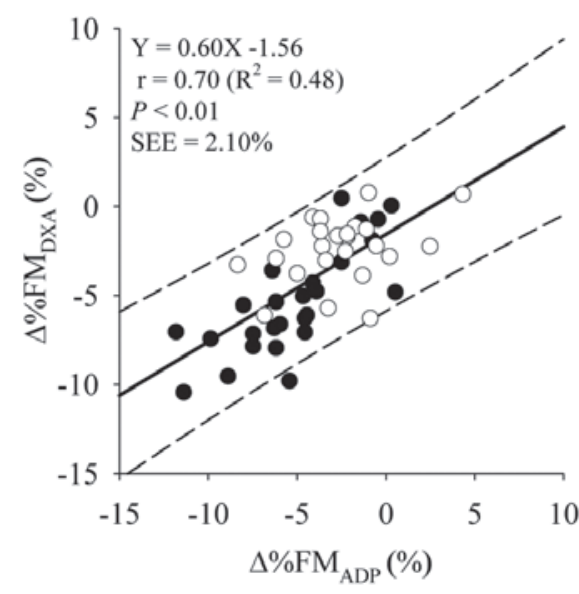

Fig. 2. BlandAltman plots for the systematic bias in the estimation of changes $(\Delta)$ in $\% F M$ between DXA and ADP. The middle solid line indicates the mean difference between $\Delta \% \mathrm{FM}$ from DXA and ADP. The upper and lower dashed lines represent limits of agreement $( \pm 2 \mathrm{SD}$ from the mean). participants engaged

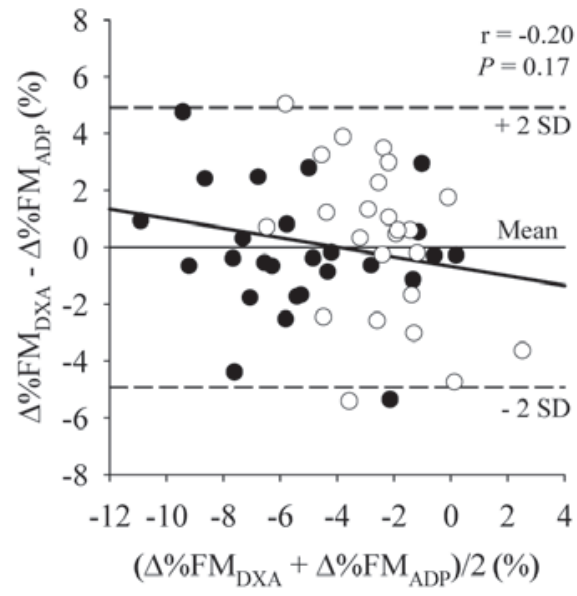

in dietary program, $\bigcirc$ : participants engaged in exercise program.
Fig. 3. Relation of changes $(\Delta)$ in $\% \mathrm{FM}$ determined with DXA minus the $\Delta \% \mathrm{FM}$ estimated by ADP to baseline \%FM determined by ADP (left) and the $\Delta \%$ FM determined by ADP (right). The middle solid line indicates the mean difference between $\Delta \% \mathrm{FM}$ estimated from DXA and ADP. The upper and lower dashed lines represent limits of agreement ( \pm 2 SD from the mean). ๑: participants engaged in dietary program, $\bigcirc$ : participants engaged in exercise program.

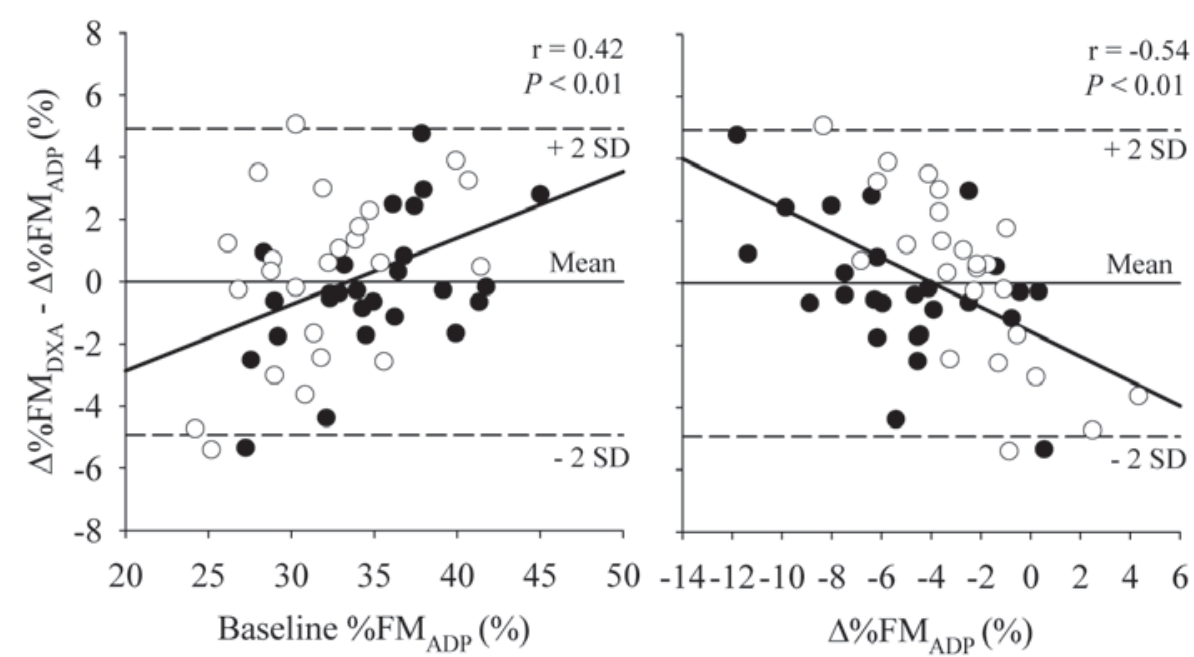

Bland-Altman plots demonstrated no significant bias in \%FM at both time points.

Figure 1 displays the results of simple regression analyses for $\Delta \% \mathrm{FM}$ by DXA and ADP. The $\mathrm{R}^{2}$ coefficients for $\Delta \% \mathrm{FM}$ were moderate $(\geq 0.4)$. Almost all plots for $\Delta \% \mathrm{FM}$ were within the $95 \%$ prediction intervals.

Figure 2 depicts the results of the Bland-Altman plots for assessing bias in the estimation of $\Delta \% \mathrm{FM}$ between the two methods. The plots demonstrated no significant bias in $\Delta \% \mathrm{FM}$. In addition, almost all individual plots for $\Delta \% \mathrm{FM}$ in both programs were within the $95 \%$ limits of agreement.

Figure 3 shows relation of $\Delta \% \mathrm{FM}$ determined with DXA minus $\Delta \% \mathrm{FM}$ estimated from ADP to baseline \%FM determined by ADP and the $\Delta \% \mathrm{FM}$ determined by ADP. ADPderived $\% \mathrm{FM}$ at baseline proved to be positively related to the differences between $\Delta \% \mathrm{FM}$ by DXA and ADP $(\mathrm{r}=0.42$, $\mathrm{p}<0.01)$. Similarly, ADP-derived $\Delta \% \mathrm{FM}$ was inversely associated with the difference $(\mathrm{r}=-0.54, \mathrm{p}<0.01)$. 


\section{Discussion}

The present study examined whether ADP could track body composition changes with weight loss in middle-aged Japanese men using DXA as the reference method. We found that ADP, using Siri's equations, tracked $\Delta \% \mathrm{FM}$ in a manner similar to that derived by DXA. We also demonstrated a uniform distribution of measurement errors (DXA minus ADP) in $\Delta \% \mathrm{FM}$ across the range of $\Delta \% \mathrm{FM}$ in the Bland-Altman analysis. However, we also showed that \%FM from individuals with larger $\% \mathrm{FM}$ by $\mathrm{ADP}$ and/or with greater $\Delta \% \mathrm{FM}$ is overestimated as compared to the DXA method.

To date, few studies have examined the ability of ADP to accurately estimate body composition change over a period of weight loss [11-13]. In 22 obese men and women with a mean BMI of $30 \mathrm{~kg} / \mathrm{m}^{2}$, Weyers et al. [11] reported no significant mean differences in $\triangle \% \mathrm{FM}$ between DXA and ADP using Siri's equation after an 8-week moderately energy-restricted diet. The $\mathrm{R}^{2}$ between DXA and ADP in their study was 0.44 for $\Delta \% \mathrm{FM}(\mathrm{p}<0.05)$. Frisard et al. [12] also reported that $\mathrm{R}^{2}$ between DXA and ADP exhibited $0.24-0.28$ for $\Delta \%$ FM (Brozek's and Siri's equations) in 56 obese men and women. Elberg et al. [20] revealed good agreement of $\Delta \% \mathrm{FM}$ between DXA and ADP using Siri's equation in natural changes over 1 year in 86 boys and girls $\left(\mathrm{R}^{2}=0.59\right)$. Moreover, a recent study of Minderico et al. [13] demonstrated no significant differences in $\Delta \% \mathrm{FM}$ between DXA and ADP in 93 obese women. They described an $\mathrm{R}^{2}$ of 0.76 for $\Delta \% \mathrm{FM}$ between DXA and ADP. The current study also demonstrated no significant differences in $\Delta \% \mathrm{FM} . \mathrm{R}^{2}$ between DXA and ADP was 0.48 for $\Delta \% \mathrm{FM}(\mathrm{p}<0.01)$. Our results are consistent with these prior studies.

Of the four previous studies, two examined the presence of systematic bias in the estimation of $\Delta \% \mathrm{FM}$ by using a Bland-Altman approach. Minderico et al. [13] indicated no significant bias, suggesting the ability of ADP to assess $\Delta \% \mathrm{FM}$ against the widespread variations ( $\mathrm{p}=0.67 ; \mathrm{r}$ value not shown). In contrast, Elberg et al. [20] reported that ADP underestimated $\Delta \% \mathrm{FM}$ as the $\% \mathrm{FM}$ gain was increased in children $(\mathrm{r}=0.35, \mathrm{p}<0.01)$. In the current study, we found no significant bias in $\triangle \% \mathrm{FM}$ in the Bland-Altman approach, suggesting the ability of ADP to track \%FM ( $r=0.20, p=$ $0.17)$. These mixed results might be accounted for by the amount of $\Delta \% \mathrm{FM}$ during follow-up periods. Along with Minderico et al. [13], we conducted weight loss intervention studies that produced large $\Delta \% \mathrm{FM}$ (mean changes of greater than 3\%) while Elberg et al. [20] detected less than $1 \%$ of $\Delta \%$ FM. Similar results were also found when comparing DXA to bioelectrical impedance, potassium counting and deuterium dilution $[12,21]$. However, our data also suggest that \%FM from individuals with larger \%FM by ADP and/ or with greater $\Delta \% \mathrm{FM}$ by ADP are overestimated as compared to the DXA method. Thus, results of similar interventions might be interpreted with caution. To our knowledge, this is the first study assessing the ability of ADP to detect $\triangle \% \mathrm{FM}$ in Asian populations using DXA as the reference method. Asians have a generally greater \%FM than Caucasians for the same BMI and age [14]. Therefore, it is important to examine the validity of ADP for body composition change in Asian populations.

It is worth addressing the advantages of ADP in research, clinical and public health settings. ADP can measure a large number of participants and a wide range of participant types. It also has the advantages of lower initial investment and no radiation exposure, even though the radiation exposure of DXA is extremely low. Since the current study revealed that ADP can track $\Delta \% \mathrm{FM}$ with no significant bias, ADP can be applied to longitudinal intervention studies around body composition to estimate $\Delta \% \mathrm{FM}$ as well as weight management strategies in clinical or public health settings. However, researchers and practitioners should consider the possibility of a $\pm 2 \%$ standard error of estimate in the estimation of $\Delta \% \mathrm{FM}$ when the participants experienced approximately $7 \%$ of weight loss.

This study has several strengths to address. First, the study population was Asian men. Previous studies with similar designs all included mainly Caucasians men and women [11-13], whereas our study enrolled a Japanese population. Our results were mostly consistent with previous studies; thus the ethnic difference may not contribute to, or hinder, the ability of ADP to track body composition changes. Second, this study elicited enough weight loss to investigate the study purpose. Previous studies with similar designs produced 4.7-7.0\% weight loss [11-13]. Our study also obtained $7.0 \%$ weight loss (approximately $10 \%$ weight loss in the dietary program), enabling us to utilize one of the widest ranges of weight loss of all published studies.

However, there were also several limitations. First, we did not validate ADP compared to the multicompartment models. Obviously, DXA is not a gold standard and seems to include certain limitations such as an ability to detect lean tissue mass changes in regions with large areas of bone pixels. In addition to this, according to the previous studies [22, 23] weight loss resulted in slight (1-2\%) reduction in hydration fraction of fat-free mass. This would potentially increase \%FM determined by DXA. However, Nord and Payne [24] reported that DXA was relatively insensitive to tissue hydration status compared to other clinical methods using the classical two-compartment model. Taken together, it is difficult to dismiss the possibility that DXA itself could be the cause of the observed bias. To correct the bias, further investigations using multicompartment models would be necessary. Second, we were unable to consider any gender-specific or age-related differences since our study recruited only middle-aged men. Therefore, it remains unclear whether our results can be extrapolated to women and older adults. In addition, we acknowledge our relatively small sample size as a limitation. 
In summary, the current study indicated that the mean changes in \%FM were similar with the two methods investigated. After an approximately $7 \%$ weight loss in obese Japanese men, DXA- and ADP-derived $\Delta \% \mathrm{FMs}$ were highly correlated. In the Bland-Altman approach, measurement errors (DXA minus $\mathrm{ADP}$ ) in $\triangle \% \mathrm{FM}$ were uniformly distributed across the ranges of body composition changes. However, $\% \mathrm{FM}$ from individuals with larger \%FM by ADP and/or with greater $\Delta \% \mathrm{FM}$ might be overestimated as compared to DXA method.

\section{Acknowledgements}

We thank all of the participants who so generously gave their time and effort toward this work. We thank Mr. Yu Okumura for his assistance with body composition measurements. This work was financially supported in part by the Tsukuba Advanced Research Alliance (TARA) Center at the University of Tsukuba (2004-2006, Tanaka Project), the 21st century COE program from the Japanese Ministry of Education, Culture, Sports, Science and Technology (2002-2006, Nishihira Project), and a grant-in-aid from the Japanese Ministry of Health, Labour and Welfare (2005-2007, Kita Project).

\section{Disclosure}

None of the authors had a conflict of interest regarding any aspect of this work.

\section{References}

1 Caterson ID, Hubbard V, Bray GA, Grunstein R, Hansen BC, Hong Y, Labarthe D, Seidell JC, Smith SC: Prevention Conference VII: Obesity, a worldwide epidemic related to heart disease and stroke: Group III: worldwide comorbidities of obesity. Circulation 2004;110:e476-e483.

$>_{2}$ Yoshiike N, Seino F, Tajima S, Arai Y, Kawano M, Furuhata T, Inoue S: Twenty-year changes in the prevalence of overweight in Japanese adults: the National Nutrition Survey 1976-95. Obes Rev 2002;3:183-190.

3 Ishikawa-Takata K, Ohta T, Moritaki K, Gotou T, Inoue S: Obesity, weight change and risks for hypertension, diabetes and hypercholesterolemia in Japanese men. Eur J Clin Nutr 2002;56:601-607.

$>_{4}$ Cui R, Iso H, Toyoshima H, Date C, Yamamoto A, Kikuchi S, Kondo T, Watanabe Y, Koizumi A, Wada Y, Inaba Y, Tamakoshi A: Body mass index and mortality from cardiovascular disease among Japanese men and women: the JACC study. Stroke 2005;36:1377-1382.

5 Roubenoff R, Kehayias JJ, Dawson-Hughes B, Heymsfield SB: Use of dual-energy X-ray absorptiometry in body-composition studies: not yet a 'gold standard'. Am J Clin Nutr 1993;58:589-591.

6 Wellens R, Chumlea WC, Guo S, Roche AF, Reo NV, Siervogel RM: Body composition in white adults by dual-energy x-ray absorptiometry, densitometry, and total body water. Am J Clin Nutr 1994;59:547-555.

7 Kohrt WM: Preliminary evidence that DEXA provides an accurate assessment of body composition. J Appl Physiol 1998;84:372-377.

8 Evans EM, Saunders MJ, Spano MA, Arngrimsson $\mathrm{SA}$, Lewis RD, Cureton KJ: Body-composition changes with diet and exercise in obese women: a comparison of estimates from clinical methods and a 4-component model. Am J Clin Nutr 1999;70:5-12.
9 McCrory MA, Gomez TD, Bernauer EM, Molé PA: Evaluation of a new air displacement plethysmograph for measuring human body composition. Med Sci Sports Exerc 1995;27:1686-1691.

10 Fields DA, Goran MI, McCrory MA: Body-composition assessment via air-displacement plethysmography in adults and children: a review. Am J Clin Nutr 2002;75:453-467.

11 Weyers AM, Mazzetti SA, Love DM, Gómez AL, Kraemer WJ, Volek JS: Comparison of methods for assessing body composition changes during weight loss. Med Sci Sports Exerc 2002;34:497-502.

12 Frisard MI, Greenway FL, Delany JP: Comparison of methods to assess body composition changes during a period of weight loss. Obes Res 2005;13: 845-854.

13 Minderico CS, Silva AM, Teixeira PJ, Sardinha LB, Hull HR, Fields DA: Validity of air-displacement plethysmography in the assessment of body composition changes in a 16-month weight loss program. Nutr Metab (Lond) 2006;3:32.

14 Levitt DG, Heymsfield SB, Pierson RN, Shapses SA, Kral JG: Physiological models of body composition and human obesity. Nutr Metab (Lond) 2009;6:7.

15 The Examination Committee of Criteria for 'Obesity Disease' in Japan; Japan Society for the Study of Obesity: New criteria for 'obesity disease' in Japan. Circ J 2002;66:987-992.

16 Kuzuya T: Prevalence of diabetes mellitus in Japan compiled from literature. Diabetes Res Clin Pract 1994;24(suppl):S15-S21.

17 Sakata K, Labarthe DR: Changes in cardiovascular disease risk factors in three Japanese national surveys 1971-1990. J Epidemiol 1996;6:93-107.
18 Siri WE: Body composition from fluid spaces and density: analysis of methods. 1961. Nutrition 1993; 9:480-491.

19 Bland JM, Altman DG: Statistical methods for assessing agreement between two methods of clinical measurement. Lancet 1986;1:307-310.

20 Elberg J, McDuffie JR, Sebring NG, Salaita C, Keil M, Robotham D, Reynolds JC, Yanovski JA: Comparison of methods to assess change in children's body composition. Am J Clin Nutr 2004;80:64-69.

21 Pødenphant J, Gotfredsen A, Engelhart M, Andersen V, Heitmann BL, Kondrup J: Comparison of body composition by dual energy X-ray absorptiometry to other estimates of body composition during weight loss in obese patients with rheumatoid arthritis. Scand J Clin Lab Invest 1996;56:615625.

22 Jebb SA, Siervo M, Murgatroyd PR, Evans S, Frühbeck G, Prentice AM: Validity of the legto-leg bioimpedance to estimate changes in body fat during weight loss and regain in overweight women: a comparison with multi-compartment models. Int J Obes 2007;31:756-762.

23 Ritz P, Sallé A, Audran M, Rohmer V: Comparison of different methods to assess body composition of weight loss in obese and diabetic patients. Diabetes Res Clin Pract 2007;77:405-411.

24 Nord RH, Payne RK: Dual-energy X-ray absorptiometry vs underwater weighing comparison of strengths and weaknesses. Asia Pac J Clin Nutr 1995;4:173-176. 